\title{
Análisis del proceso de toma de decisiones estratégicas de gestión contable en una empresa y su influencia en las políticas de calidad en la industria ecuatoriana: un estudio de caso
}

\section{Analysis of the strategic decision making process of accounting management in a company and its influence on quality policies in the Ecuadorian industry: a case study}

Santiago Nájera, $\mathrm{PhD}$.

Universidad Internacional del Ecuador, Ecuador

David Hernán Montenegro Moreno, MBA

Pontificia Universidad Católica del Ecuador, Ecuador

María Auxiliadora Guerrero Bejarano, MBA

Universidad Internacional del Ecuador, Ecuador

Autor por correspondencia: sanajera@uide.edu.ec,dhmontenegro@yahoo.es, maguerrerobe@uide.edu.ec

Fecha de recepción: 21 de Febrero de 2018 - Fecha de aceptación: 15 de agosto de 2018

\section{Resumen}

El proceso de toma de decisiones estratégicas de gestión contable en una empresa y su influencia en las políticas de calidad constituye un tema estratégico clave para ser estudiado y el método de estudio del caso es una alternativa válida para efectuar una investigación cualitativa que permita obtener una comprensión significativa del caso específico.

Palabras Claves: gestión contable; estrategia; estudio del caso

\begin{abstract}
The process of making strategic decisions on accounting management in a company and its influence on quality policies is a key strategic issue to be studied and the case study method is a valid alternative to carry out qualitative research to obtain an understanding significant of the specific case.
\end{abstract}

Key words: accounting management; strategy; study of the case 


\section{Introducción}

Una metodología apropiada para el estudio concreto de un caso único es el método de estudio del caso (Yin 2015). Método que se halla vinculado a la investigación netamente cualitativa y tiene un enfoque orientado al alcance de una comprensión significativa del caso, a través de la realización de un exhaustivo análisis, vinculado en la presente casuística al proceso de toma de decisiones estratégicas de gestión contable y de su influencia en las políticas de calidad de la empresa objeto de estudio.

Aun cuando en una organización todos sus miembros toman decisiones, este proceso es más importante en lo que se relaciona con el trabajo del gerente (Noda and Bower 1996). Los gerentes son quienes toman las decisiones al momento de planificar, organizar, dirigir o controlar en una empresa, es decir en el marco del proceso administrativo (Robbins, 2010). Estas decisiones influyen en varios aspectos empresariales, entre ellos las políticas de calidad. Las decisiones gerenciales se enfocan en temas sociales, humanos, económicos, etc. En este sentido, la contabilidad de gestión puede constituir una herramienta importante, ya que provee elementos a los gerentes para la toma de decisiones empresariales.

\section{Marco teórico}

Uno de los temas más importantes que influye sobre la toma de decisiones constituye el liderazgo de las cabezas de la organización (Mintzberg 1990). En este sentido, es claro que en los equipos con mayor cantidad participantes existe más dificultad para llegar a un consenso; a diferencia de los equipos con inferior número de miembros, en donde llegar a un consenso se vuelve tarea más fácil (Radomska 2014). También es preponderante el papel de la alta gerencia, de acuerdo con investigaciones efectuadas, sus decisiones o propuestas difícilmente son rebatidas o confrontadas por quienes poseen un nivel jerárquico menor. (Lussier R. \& Achua C., 2002). De este modo, resultaría más conveniente que en el equipo que toma las decisiones exista una estructura más horizontal que permita y haga factible un mejor intercambio de experiencias y opiniones.

La incertidumbre, la ambigüedad y las cambiantes circunstancias actuales exigen que los líderes tengan el valor de tomar decisiones difíciles (Merigó 2015). Para ello existen tres perspectivas fundamentales para la toma de decisiones: la racionalidad, la racionalidad limitada y la intuición. La racionalidad se refiere a tomar decisiones de manera objetiva, definiendo desde un inicio un problema claro por resolver y una fuerte orientación hacia la meta para lograr resultados óptimos, considerando que los criterios y alternativas pueden definirse de acuerdo con su importancia. La racionalidad limitada difiere de la racionalidad en la medida en que no existe una orientación tan marcada hacia los resultados, ya que puede aceptarse una alternativa satisfactoria, aunque no maximice los beneficios deseados. La intuición constituye un proceso inconsciente por el cual se toman decisiones a partir de la experiencia y el juicio personal acumulado. (Robbins, 2010).

Independientemente de las perspectivas descritas, la toma de decisiones constituye un proceso. Por ende, existen pasos que se podrían seguir para poder analizar y escoger la mejor alternativa, más aún cuando confluyen factores económicos, sociales, tecnológicos que pueden 
ser clave en el momento de una decisión estratégica empresarial (Robbins, 2010). El esquema se presenta a continuación en la figura 1:
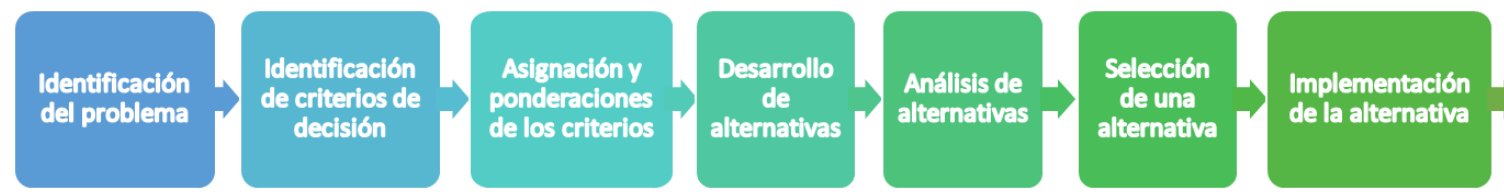

Evaluación de la

Figura 1. Esquema del proceso de toma de decisiones

Una herramienta muy útil para los gerentes a la hora de tomar decisiones constituye la contabilidad de gestión (Gond, Grubnic et al. 2012). La National Association of Accountants indica que en la contabilidad de gestión se identifican conceptos relacionados con la planificación, evaluación, control, aseguramiento del uso eficiente de los recursos y presentación de informes externos. (Belkaoui, 1992). Se considera que el objetivo básico de la contabilidad de gestión es asistir al gerente en la toma de decisiones. En este sentido, las principales características cuantitativas de la contabilidad de gestión son la relevancia, congruencia de objetivos, exactitud, precisión, fiabilidad, consistencia, comparabilidad, verificabilidad, flexibilidad, adaptabilidad, oportunidad, etc., (Belkaoui, 1980). Pese a ser cuantitativas, están sujetas a una interpretación y a una comprensión significativa cualitativa, ya que en última instancia, las decisiones tienen su génesis en el entendimiento cualitativo de la parte numérica. De este modo, se evidenciaría la relación entre lo contable y lo administrativo para la toma de decisiones.

\section{Método de investigación}

Cuando se utiliza el método de estudio del caso, una de las posibles fuentes de información a considerarse constituye la entrevista a profundidad semi-estructurada (Creswell 2012). En esta investigación corresponde la fuente primaria de información. Sin embargo, la información que se extrae de la misma corresponde a un proceso de múltiples recodificaciones de la información en búsqueda de obtener la esencia de la información obtenida, conforme se visualiza en la figura 2. 


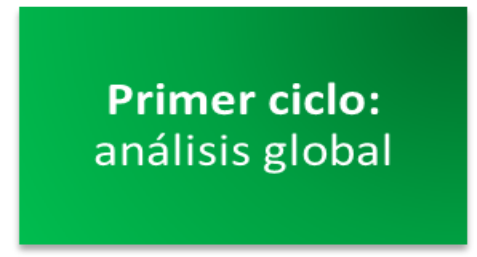

Tercer ciclo: se

recodifican nuevamente

los datos y se agrupan los códigos en familias
Segundo ciclo: tres tipos de

codificación: en vivo;

codificación manual teórica; y codificación manual

impresionista.

Cuarto ciclo: se procede a

definir las categorías de

los códigos y la

construcción de redes

semánticas

Quinto ciclo: con las redes

semánticas entorno a los

códigos y/o categorías, se

genera el modelo emergente

que busca describir la realidad

de los datos analizados.

Figura 2. Proceso de codificación y recodificación.

Con la finalidad de efectuar una adecuada validación de los datos obtenidos es recomendable realizar la triangulación de datos. Esta técnica permite verificar si el trabajo de investigación realizado está dentro de los parámetros correctos (Penalva Verdú, Alaminos Chica et al. 2015). La intención es que el investigador pueda asegurar la validez de los resultados de sus mediciones, contrastando con otro tipo de información, como por ejemplo reportes de ventas, manuales de calidad, declaraciones tributarias, hojas de control de producción, análisis financieros, etc., en fin, cualquier dato que permita cotejar lo recabado en las entrevistas con información real. Adicionalmente, es importante recordar que en el estudio de casos se debe establecer un significado, no una posición; se pretende llegar a una interpretación, la cual debe ser revisada a fin de asegurar que los datos sean precisos y contribuyan a los objetivos de la investigación (Stake, 1999).

\section{Resultados}

En un estudio efectuado en una empresa textil ecuatoriana, en donde se utilizó el método de estudio del caso, se evidenció que su estrategia es enfocada fundamentalmente en las políticas de calidad, es decir en otorgar productos de calidad que satisfagan enteramente las necesidades y requerimientos de los clientes.

En lo referente a la toma de decisiones se evidenció que cualquier decisión se la toma de manera consensuada entre los miembros de esta empresa familiar, previa la realización de un análisis costo beneficio con enfoque financiero para evaluar la viabilidad de realizar una inversión. La empresa cuenta además con un equipo de trabajo multidisciplinario, ya que existe un ingeniero electrónico, un ingeniero mecánico y un profesional contable y financiero, lo que permite una interacción apropiada para poder tomar decisiones basadas en criterios técnicos y análisis de costos. Otro de los aspectos que la empresa considera para la toma de decisiones es el 
entorno económico, social y tecnológico, ya que estos factores influyen de manera directa en las actividades empresariales.

En lo que respecta a la toma de decisiones estratégicas de gestión contable, se pudo evidenciar que el equipo multidisciplinario de la empresa considera elementos inherentes al ámbito financiero y contable, tales como presupuestos, análisis de costos, análisis de los proveedores, detección de proveedores que sean empresas fantasmas o inexistentes. Empresas fantasmas se consideran aquellas que se han constituido mediante una declaración ficticia de voluntad o con ocultación deliberada de la verdad, quienes, fundadas en el acuerdo simulado, aparentan la existencia de una sociedad, empresa o actividad económica, para justificar supuestas transacciones, ocultar beneficios, modificar ingresos, costos y gastos o evadir obligaciones (Art. 25 del Reglamento de Aplicación a la Ley de Régimen Tributario Interno). Empresas inexistentes son aquellas cuyo proceso productivo y comercial, así como su constitución, no ha sido verificada. (Art. 24 del Reglamento de Aplicación a la Ley de Régimen Tributario Interno). En este sentido, se evidenció que en la empresa se realizan estas consideraciones inherentes a temas contables y tributarios para tener insumos que permitan definir estrategias de gestión contable relacionadas con la maximización de beneficios, como por ejemplo inversión en tecnología de punta y en materia prima de calidad.

En dicho estudio se pudo apreciar que las estrategias están enfocadas al cumplimiento de los objetivos y son generadas por parte de cada uno de los miembros del equipo multidisciplinario, sin dejar de considerar las opiniones y sugerencias del personal operativo. Las estrategias se consolidan en el marco del consenso de un equipo multidisciplinario. Es imprescindible destacar que la calidad se la entiende como un todo, es decir que cada esfuerzo o actividad efectuada tiende a influir en la calidad, en sus políticas, partiendo desde la Alta Gerencia que constituye el génesis de la estrategia. Las decisiones estratégicas relacionadas con la contabilidad de gestión están enfocadas en la maximización de los beneficios a través del mejoramiento de procesos, de la adquisición de tecnología de punta y materia prima de calidad.

De igual forma en tener un exhaustivo control de los proveedores a fin de asegurar que éstos no tengan falencias en la calidad o peor aún en su esencia y comportamiento ético empresarial (empresas fantasmas o inexistentes). Por ende, es claro que para la obtención de un producto final sin fallas, resulta necesario tener un alto nivel de compromiso de la alta gerencia y un buen manejo y enfoque de contabilidad de gestión para tomar las mejores decisiones estratégicas.

\section{Conclusiones}

El método de estudio del caso es muy recomendable dentro de la investigación cualitativa, ya que permite verificar de manera directa la realidad de un caso específico y concreto, además de contrastar la información obtenida con la realidad. Si bien, los resultados que se obtienen de la investigación, al tratarse de un caso único, no son generalizables, sí es factible obtener una comprensión significativa del estudio efectuado estableciendo significados. Esto puede servir como base, para en un futuro efectuar estudios basados en esta metodología para varias empresas, para de este modo formarse como investigador un criterio más amplio de un tema específico, como puede ser por ejemplo el análisis del proceso de toma de decisiones estratégicas de gestión contable en una empresa y su influencia en las políticas de calidad. 


\section{Bibliografía}

Álvarez C. \& San Fabián J. (2012). La elección del estudio de caso en investigación educativa. Obtenido de http://www.ugr.es/ pwlac/G28_14Carmen_AlvarezJoseLuis_SanFabian.html

Álvarez C., B. E. (2010). Una valoración de la estrategia, a través de la innovación, en organizaciones de Guanajuato, México. Obtenido de Avolio, B. (2010). Métodos Cualitativos de Investigación: Una aplicación al estudio de caso. México: Cengage Learning.

Bateman, T., \& Snell, S. (2004). Administración de una ventaja competitiva. México: Mc Graw Hill.

Belkaoui, A. (1980). Conceptual Foundations of Management Accounting.

Belkaoui, A. (1992). The New Foundations of Management Accounting. New York. Quorum Books.

Creswell, J. (2012). Qualitative Inquiry and Research Design: Choosing Among Five Approaches, SAGE Publications.

Deming, W. (1989). Calidad, productividad y competitividad: la salida de la crisis. México: Ediciones Díaz de Santos.

Gallardo, J. (2012). Administración estratégica de la visión a la ejecución. México: Alfaomega Grupo Editor.

Gond, J.-P., et al. (2012). "Configuring management control systems: Theorizing the integration of strategy and sustainability." Management Accounting Research 23(3): 205-223.

Ishikawa, K. (1994). Introducción al control de la calidad. México: Diaz de Santos. 86

ISO 9001, S. G. (2008). Norma Internacional Sistema de Gestión de Calidad ISO 9001. Ginebra.

Juran, J. (2005). Critical Evaluations in Business and Management. New York: Psychology Press.

Lussier R. \& Achua C. (2002). Liderazgo, Teoría, Aplicación, Desarrollo de Habilidades: Thomson Learning.

Maxwell, J. (1996). Qualitative Research Design. An Interactive Approach. London: Sage Publications.

Méndez, M., \& Reyes, A. (2004). Análisis financiero y mejoramiento de procesos productivos de una fábrica de medias, caso: Reyes Industria Textil CIA. LTDA (tesis de pregrado). Quito.

Merigó, J. M. (2015). "Decision-making under risk and uncertainty and its application in strategic management." Journal of Business Economics and Management 16(1): 93-116. 
Mintzberg, H. A. (1999). Estrategía Safari:Una visita guiada por la selva de la Gestión Estrategica: Simon and Schuster. En Strategy Safari.

Mintzberg, H. (1990). "The manager's job: Folklore and fact." Harvard business.

Noda, T. and J. L. Bower (1996). "Strategy making as iterated processes of resource allocation." Strategic Management Journal 17(S1): 159-192.

Patton, M. (1990). Qualitative evaluation and research methods. En K. Knafl, Designing Qualitative Studies (págs. 169-86). Beverly Hills, CA: Sage.

Penalva Verdú, C., et al. (2015). "La investigación cualitativa: técnicas de investigación y análisis con Atlas. ti."

Phillips, L. (1998). Combining quantitative and qualitative approaches to social research in human geography, an impossible mixture? Environment and planning A, 30(2), 261-76.

Porter, M. (1980). Competitive Strategy: Techniques for Analyzing Industries and Competitors. New York.

Porter, M. (1996). What is Strategy? Harvard Business Review, November-December.

Radomska, J. (2014). "Linking the Main Obstacles to the Strategy Implementation with the Company's Performance." Procedia - Social and Behavioral Sciences 150(0): 263-270.

Reglamento de Aplicación a la Ley de Régimen Tributario Interno, R.O. 448, 28 de febrero de 2015

Robbins, S. (2010). Administración. México: Pearson Education.

Stake, R. (1999). Investigación con estudio de casos. Madrid: Ediciones Morata.

Summers, D. (2006). Administración de la calidad. México: Pearson Education.

Thompson, A., \& Strickland, A. (2001). Administración Estratégica. México: Mc Graw Hill.

Thompson A., Peteraf M., Gamble J., Strickland A., (2012). Administración Estratégica. México: Mc Graw Hill.

Yin, R. (2009). Case Study Research, Sage. E.E.U.U.

Yin, R. K. (2015). Qualitative Research from Start to Finish, Guilford Publications.

Creswell, J. (2012). Qualitative Inquiry and Research Design: Choosing Among Five Approaches, SAGE Publications. 
Gond, J.-P., et al. (2012). "Configuring management control systems: Theorizing the integration of strategy and sustainability." Management Accounting Research 23(3): 205-223.

Merigó, J. M. (2015). "Decision-making under risk and uncertainty and its application in strategic management." Journal of Business Economics and Management 16(1): 93-116.

Mintzberg, H. (1990). "The manager's job: Folklore and fact." Harvard business.

Noda, T. and J. L. Bower (1996). "Strategy making as iterated processes of resource allocation." Strategic Management Journal 17(S1): 159-192.

Penalva Verdú, C., et al. (2015). "La investigación cualitativa: técnicas de investigación y análisis con Atlas. ti."

Radomska, J. (2014). "Linking the Main Obstacles to the Strategy Implementation with the Company's Performance." Procedia - Social and Behavioral Sciences 150(0): 263-270.

Yin, R. K. (2015). Qualitative Research from Start to Finish, Guilford Publications. 